medgen 2018 · 30:429-437

https://doi.org/10.1007/s11825-018-0214-2

Online publiziert: 8. Januar 2019

(c) Der/die Autor(en) 2018

CrossMark

\author{
Oliver Gross ${ }^{1}$. Julia Hoefele ${ }^{2}$ \\ ${ }^{1}$ Klinik für Nephrologie und Rheumatologie, Universitätsmedizin Göttingen, Göttingen, Deutschland \\ ${ }^{2}$ Institut für Humangenetik, Klinikum rechts der Isar, Technische Universität München, München, \\ Deutschland
}

\title{
Genetische Ursachen und Therapie beim Alport-Syndrom
}

Innenohrschwerhörigkeit sowie okuläre Veränderungen (z.B. Lentikonus anterior) entwickeln [1].

In den letzten Jahren hat das Verständnis der pathophysiologischen Grundlagen von Nierenerkrankungen deutlich zugenommen. Für viele Nierenerkrankungen, die im Kindes- und jungen Erwachsenenalter manifest werden, konnte eine hereditäre Ursache nachgewiesen werden. Die Kenntnis der genetischen Ursache ist bei vielen dieser Erkrankungen inzwischen für die Therapie und damit für deren klinischen Verlauf entscheidend. Eine interdisziplinäre $\mathrm{Zu}$ sammenarbeit von (Kinder-)Nephrologen und Humangenetikern bei der Betreuung dieser Patienten spielt daher eine wichtige Rolle. Am Beispiel des AlportSyndroms (AS) soll dies im Folgenden näher erläutert werden.

\section{Symptomatik beim Alport- Syndrom}

Das AS ist gekennzeichnet durch Hämaturie und Proteinurie, bedingt durch strukturelle Veränderungen in der glomerulären Basalmembran (GBM) mit charakteristischer Aufsplitterung und Lamellierungen, und führt unbehandelt im jungen Erwachsenenalter zu terminalem Nierenversagen. Mittel- bis langfristig entwickelt sich eine glomeruläre und interstitielle Fibrose. Circa 1-2 \% der Patienten mit terminalem Nierenversagen in westlichen Ländern weisen ein AS auf. Patienten mit AS können neben der progredienten Niereninsuffizienz auch eine

\section{Mehr Informationen zum Thema}

Patientengruppe http://www.alportselbsthilfe.de

\section{Genetische Grundlagen beim Alport-Syndrom}

Beim AS handelt es sich um eine heterogene Erkrankung, bedingt durch Varianten in Genen, die für Kollagen Typ IV kodieren. Sechs Gene (COL4A1-COL4A6), entsprechend der sechs homologen Kollagen-Ketten ( $\alpha 1-\alpha 6)$ für Kollagen Typ IV, sind bekannt. Ursächlich für AS sind Varianten in den Genen COL4A3, COL4A4 und COL4A5 (• Tab. 1; [2]). Kollagen Typ IV ist Hauptbestandteil aller Gefäßbasalmembranen. An mechanisch besonders beanspruchten Stellen wie der GBM, dem Innenohr und der Augenlinse besteht das Typ IV Kollagennetzwerk aus a3.a4.a5-Ketten, die eine sogenannte Triple-Helix-Struktur bilden. Es kommt hierbei zu einer engen Verdrillung der Kollagenketten, was durch das Vorhandensein von Glycin an jeder dritten Aminosäureposition bedingt ist. Ein Austausch von Glycin führt zum Abknicken der Triple-Helix-Struktur, trunkierende Varianten zu einem vorzeitigen Kettenabbruch. Bei bis zu $95 \%$ der Patienten können in den bisher bekannten Genen COL4A3, COL4A4 und COL4A5 ursächliche Varianten nachgewiesen werden, wobei es sich meist um Glycin-, Nonsense-, Splice-Site- und Frameshift-Varianten sowie größere Deletionen bzw. Duplikationen handelt [3]. Die Einschätzung anderer Varianten erscheint derzeit noch problematisch, ggf. handelt es sich in diesen Fällen um Varianten ohne oder mit unklarer klinischer Relevanz. Hier ist die Anwendung elektronischer Vorhersageprogramme hilfreich. Auch die Einschätzung bei Identifikation mehrerer Varianten ist aktuell noch schwierig, klare Empfehlungen können diesbezüglich daher nicht gegeben werden. Mehrere Erbgänge können beim AS unterschieden werden: (1) der X-chromosomale (Variante im COL4A5-Gen), (2) der autosomale (autosomal-rezessiv und autosomal-dominant; Variante(n) im COL4A3oder COL4A4-Gen) und (3) der digenische (Varianten im COL4A3-, COL4A4und COL4A5-Gen) $[1,4,5]$. Die Häufigkeit des X-chromosomalen AS wird auf 1:5000 geschätzt, die Prävalenz des autosomal-rezessiven AS auf 1:50.000, der autosomal-dominante sowie der digenische Erbgang sind deutlich seltener. Es wird vermutet, dass - zumindest für COL4A5 - bisher nur ca. $10 \%$ der ursächlichen Varianten in der Literatur bekannt sind [3], Angaben für die Gene COL4A3 und COL4A4 liegen bisher nicht vor. Fast $1 \%$ der Bevölkerung sind heterozygote Anlageträger für Varianten in den Genen COL4A3 und COL4A4 [6]. Die Verteilung von Varianten in den Genen COL4A3 und COL4A4 ist ungefähr gleich. Für die X-chromosomale Form ist eine Genotyp-Phänotyp-Korrelation bekannt [7]. Durch Inframe- oder Missense-Varianten werden Veränderungen in der Proteinstruktur hervorgerufen, die sich meist in einem milderen Verlauf der Erkrankung mit späterem Beginn der terminalen Niereninsuffizienz widerspiegeln (terminales Nierenversagen unbehandelt mit Beginn $<40$ Jahren). Frameshift-, Nonsense- und große Rearrangement-Varianten hingegen führen 
Tab. 1 Genetische Ursachen des Alport-Syndroms (AS) und der "thin basement membrane nephropathy" (TBMN)

\begin{tabular}{|l|l|l|l} 
& COLA3 und COL4A4 & COLA5 \\
\hline Chromosom & 2q36.3 & Xq22.3 \\
\hline Erbgang & $\begin{array}{l}\text { autosomal-rezessiv } \\
\text { autosomal-dominant } \\
\text { digen }\end{array}$ & $\begin{array}{l}\text { X-chromosomal } \\
\text { digen }\end{array}$ \\
\hline Häufigkeit & $\begin{array}{l}10 \% \text { autosomal-rezessiv und -dominant } \\
\text { selten digen }\end{array}$ & $\begin{array}{l}85 \% \\
\text { selten digen }\end{array}$ \\
\hline Prävalenz & $\begin{array}{l}1: 50.000 \text { autosomal-rezessiv } \\
\text { selten autosomal-dominant und digen }\end{array}$ & $\begin{array}{l}1: 5000 \\
\text { selten digen }\end{array}$ \\
\hline Klinische Präsentation & $\begin{array}{l}\text { AS } \\
\text { TBMN }\end{array}$ & AS \\
\hline
\end{tabular}

zur Verkürzung des Proteins und folglich schwerem Verlauf der Erkrankung mit frühem Beginn der terminalen Niereninsuffizienz (terminales Nierenversagen unbehandelt mit Beginn <30 Jahren). Patienten mit Splice-Site- oder trunkierenden Varianten oder mit Varianten, die am $5^{\prime}$-Ende des Gens lokalisiert sind, weisen ein deutlich erhöhtes Risiko für eine extrarenale Beteiligung (Augenveränderungen, Innenohrschwerhörigkeit) auf.

Digene und biallelische Vererbung die COL4A3-5-Gene betreffend wurde in Einzelfällen bei AS in der Literatur beschrieben. Bezüglich des Phänotyps konnte zumindest für Patienten mit einer heterozygoten Variante in COL $4 A 3$ und COL4A4 ein leichterer Phänotyp im Vergleich zu Patienten mit zwei heterozygoten Varianten in COL4A3 oder COL4A4 beobachtet werden. Zudem ist ein leichterer Phänotyp bei Patienten mit Varianten in cis im Vergleich zu in trans festzustellen [3]. Segregationsanalysen mit Berücksichtigung des klinischen Phänotyps helfen hierbei, die einzelnen Varianten und ihre klinische Relevanz zu beurteilen.

\section{Frauen und Alport-Syndrom}

Mädchen und Frauen mit einer heterozygoten Variante in COL4A5 weisen sowohl intra- wie auch interfamiliär ein breites Spektrum an klinischen Symptomen auf, welches von Mikrohämaturie bis hin zum Vollbild des AS reicht [8]. Bis $\mathrm{zu} 30 \%$ der Frauen entwickeln bis zum 60. Lebensjahr eine Niereninsuffizienz [9]. Zusätzlich besteht ein Risiko von jeweils ca. $30 \%$, eine Hochton- schwerhörigkeit und/oder Retinopathie zu bekommen. Zudem haben Frauen im Vergleich zur Allgemeinbevölkerung ein 2- bis 3-fach erhöhtes Risiko für kardiale Ereignisse. Eine Genotyp-PhänotypKorrelation bei Frauen konnte bislang nicht nachgewiesen werden [10]. Als Ursache für die phänotypische Variabilität bei Mädchen und Frauen werden u. a. eine ungünstige $\mathrm{X}$-Inaktivierung und das Vorhandensein von genetischen Modifiern vermutet $[5,11,12]$.

\section{Differentialdiagnose}

Die Nephropathie vom Typ der dünnen Basalmembran (,thin basement membrane nephropathy", TBMN) wird mit einer Häufigkeit von ca. $1 \%$ in der Allgemeinbevölkerung angegeben und wurde früher als eigenständiges Krankheitsbild betrachtet $[6,13]$. Bei den meisten Individuen mit TBMN können heterozygote Varianten in COL4A3 oder COL4A4 nachgewiesen werden (• Tab. 1; [14]), wobei auch hier eine relative Gleichverteilung der Variantenhäufigkeit in COL4A3 bzw. COL4A4 festzustellen ist. Die TBMN ist gekennzeichnet durch Hämaturie sowie ggf. durch eine geringe Proteinurie, früher wurde sie auch als benigne familiäre Hämaturie $(\mathrm{BFH})$ bezeichnet. Das Auftreten einer Nierenfunktionseinschränkung wird bei bis zu $40 \%$ der Patienten mit einer TBMN in höherem Lebensalter beobachtet, die Entwicklung einer terminalen Niereninsuffizienz nur selten (1-2\%) [1]. Bisher sind nur relativ wenige Varianten ursächlich für TBMN in der Literatur beschrieben, Frequenzunterschiede bzgl. Varianten in COL4A3 bzw. COL4A4 sind derzeit nicht zu beobachten. Aufgrund des erhöhten Risikos einer ernsthaften Nierenerkrankung werden Patienten mit TBMN mittlerweile zum AS hinzugezählt, um Patienten mit einer heterozygoten Variante (autosomale Form) durch die Diagnose Alport-Syndrom eine lebenslange nephrologische Betreuung zu ermöglichen [15]. Daher kommt hier dem Humangenetiker mit seiner frühzeitigen genetischen Diagnose eine wichtige Lotsenfunktion $\mathrm{zu}$.

\section{Molekulargenetische Diagnostik}

Da eine klinische und histologische Unterscheidung beider Entitäten (AS, autosomal heterozygotes $\mathrm{AS} / \mathrm{TBMN}$ ) häufig schwierig ist, spielt die molekulargenetische Diagnostik, gerade auch im Hinblick auf einen frühestmöglichen Therapiebeginn bei AS, eine entscheidende Rolle. Aufgrund der Heterogenie und gerade auch hinsichtlich einer möglichen digenen Vererbung und/oder genetischer Modifier wird inzwischen empfohlen, Next Generation Sequencing (NSG)Technologien (Panel-Diagnostik, Exomund ggf. Genom-Analysen) zur molekulargenetischen Abklärung zu verwenden [16]. Ist die Variante bei einem Patienten mit AS mittels NGS identifiziert, können sich (asymptomatische) Familienangehörige mittels Zieldiagnostik (SangerSequenzierung) auf die familiäre(n) Variante(n) untersuchen lassen.

\section{Organoprotektion bei chronischer Nierenfibrose}

Die Durchführung einer molekulargenetischen Diagnostik ermöglicht die frühzeitige Diagnose noch vor Beginn der klinischen Symptomatik im Kleinkindesalter und eröffnet so die Möglichkeit einer präemptiven Therapie. COL4A3-/-Mäuse erkranken am Vollbild des AS und erlauben so mögliche Therapieoptionen [17-20]: Die präemptive Gabe von Ramipril zeigte hierbei ein deutlich verlängertes Überleben der Tiere [19]. Der Effekt ist dabei jedoch stark abhängig vom Zeitpunkt des Therapiebeginns. Ein früher Beginn der Therapie bewirkte deutlichere Effekte als 
ein später Beginn. Für AT1-Antagonisten, HMG-CoA-Reduktase-Inhibitoren und Paricalcitol konnte ebenfalls eine - allerdings geringer ausgeprägte - nephroprotektive und antifibrotische Wirkung nachgewiesen werden $[20,21]$.

\section{Aktuelle Therapiestudien und Therapieansätze}

Die Effektivität und Sicherheit von Ramipril bei Kindern in sehr frühen Krankheitsstadien wird derzeit in der deutschlandweiten EARLY PRO-TECT AlportStudie (•Tab. 2) untersucht. Die RAASBlockade beim AS hat sich dank des Therapieregisters in den letzten Jahren etabliert und gilt als sehr effektiv und bei Erwachsenen sicher.

Regulus Therapeutics führt die Phase 2-Studie HERA mit anti-microRNA21 (miR21) durch, das im AlportMausmodell über „peroxisome proliferator-activated receptor- $\alpha$ “ insbesondere die tubulointerstitielle Fibrose hemmt $[22,23]$. Reata Pharmaceuticals führt die Phase 2/3-Studie CARDINAL mit Bardoxolonmethyl durch, zu dem keine Daten aus dem Alport-Mausmodell vorliegen, aber klinische Daten zur diabetischen Nephropathie [24], die zum vorzeitigen Studienabbruch führten. Bardoxolonmethyl greift potenziell unter anderem über den NFkb-Pathway in die Pathogenese der Nierenfibrose ein [22]. Auch hier ist unklar, wie gut sich präklinische Ergebnisse auf den Men- schen übertragen lassen. Eine weitere zukünftige Therapieoption stellen Kollagenrezeptor-Inhibitoren wie Integrine sowie Discoidin Domain Rezeptor 1 (DDR1) dar. Der Verlust bzw. die Blockade von DDR1 hemmt die Produktion von Typ IV Kollagen und verlangsamt im AS-Mausmodell den Verlauf der Nierenfibrose [25-28]. Letztlich kann der dem AS zugrunde liegende Gendefekt nur durch gen- und zellbasierte Therapien geheilt werden [29-32]. Die unklare Datenlage rückt die Knochenmarktransplantation als mögliche kurative Therapie noch in weite Ferne.

\section{Nephrologischer Leitfaden zu Diagnose und Verlaufskontrolle}

\section{(1) Zeitpunkt und Methoden der Frühdiagnose: eine Domäne der Humangenetik}

Besonders im kindernephrologischen Bereich ist in Deutschland die molekulargenetische Diagnostik Goldstandard für die Diagnose von Typ IV KollagenErkrankungen und ersetzt mittlerweile oft die Nierenbiopsie. Jedes Kind, auch Mädchen mit einer heterozygoten Variante, sollte bereits im Kindesalter genetisch diagnostiziert werden, da die Diagnose therapeutische Konsequenzen hat. Wegen der Ausreifung der GBM sollte das Screening beim Kinderarzt auf Mikrohämaturie bei AS ab dem 2. Lebensjahr, spätestens bei der U8, erfolgen. Jede Mikrohämaturie renalen Ursprungs oder Mikroalbuminurie sollte zur Überweisung zum Kindernephrologen führen. Das AS ist zunächst eine klinische Diagnose des erfahrenen (Kinder-)Nephrologen, die häufig schon durch sorgfältige Untersuchung und (Familien-)Anamnese gestellt werden kann (• Abb. 1). Jeder Verdacht auf AS ist wegen der Therapiemöglichkeiten mittels vollständiger Diagnostik abzuklären. Leider liegen oft viele Jahre zwischen den ersten Symptomen und der Diagnosestellung [34] mit einem traurigen Gipfel der zu spät erkannten Fälle zwischen dem 10. und 25., teils auch 30. Lebensjahr (trotz eindeutiger Symptome wie Proteinurie und Ödemen). Jede Familie sollte humangenetisch beraten und idealerweise bereits vom Humangenetiker auf Therapiemöglichkeiten hingewiesen werden. Nierenbiopsien sind zwingend auch elektronenmikroskopisch aufzuarbeiten, typische lichtmikroskopische (Fehl-)Diagnosen sind die fokal segmentale Glomerulosklerose (FSGS) oder die mesangioproliferative Glomerulonephritis.

\section{(2) Art und Häufigkeit der Kontrolluntersuchungen, insbesondere bei Hämaturie}

Heterozygote Anlageträger des X-chromosomalen (XLAS) oder autosomalrezessiven Alport-Syndroms (ARAS) mit einer dünnen Basalmembran sind

Hier steht eine Anzeige. 
darüber aufzuklären, dass der klinische Verlauf nicht immer benigne ist. Bis $\mathrm{zu} 40 \%$ der Patienten entwickeln im höheren Alter eine Nierenfunktionseinschränkung, bis zu $20 \%$ sind auf Dialyse angewiesen. Mutmaßlich ist die Dialyse bei den heterozygoten Anlageträgern durch frühzeitige RAAS-Blockade komplett vermeidbar. Heterozygote Anlageträger sollten daher ab Diagnosestellung jährlich und lebenslang auf weitere Risikofaktoren untersucht werden. Erwachsene heterozygote Anlageträger sollten bereits ab dem Auftreten einer Mikroalbuminurie behandelt werden, Kinder erst $a b$ dem Auftreten einer Proteinurie. In späteren Stadien unterscheidet sich die Therapieeskalation nicht vom „normalen“ Patienten mit AS (s. unter Punkt 5).

Patienten, die hemizygote, compound-heterozygote oder homozygote Varianten aufweisen, entwickeln $\mathrm{zu}$ $100 \%$ eine terminale Niereninsuffizienz. Die Zeitspanne bis zur Dialyse ist abhängig vom Therapiebeginn und der Variante, Patienten mit Glycin-Missense-Varianten zeigen einen langsameren Verlauf der Erkrankung. Daher sind diese Patienten ab Diagnosestellung mindestens halbjährlich und lebenslang vom Nephrologen auf Mikroalbuminurie und Risikofaktoren zu untersuchen.

Im Erwachsenenbereich bedeutet dies, dass jeder Patient mit Mikrohämaturie glomerulären Ursprungs schriftlich über sein potenzielles Risiko aufgeklärt und jährlich beim Hausarzt auf Mikroalbuminurie untersucht werden sollte. Bei Auftreten von Mikroalbuminurie sollte sofort eine erneute nephrologische Vorstellung erfolgen. Jedes Kind mit persistierender Mikrohämaturie glomerulären Ursprungs, insbesondere bei weiteren betroffenen Familienmitgliedern, sollte frühzeitig einer genetischen Diagnostik auf AS durch den Humangenetiker zugeführt werden.

\section{(3) Optimaler Therapiebeginn}

In Beobachtungsstudien konnte gezeigt werden, dass ACE-Hemmer die Dialyse im Median um 18 Jahre verzögern und die Lebenserwartung verbessern [35]. Die nachfolgende Empfehlung (• Abb. 2) gibt

medgen 2018·30:429-437 https://doi.org/10.1007/s11825-018-0214-2

(c) Der/die Autor(en) 2018

\section{O. Gross $\cdot$ J. Hoefele}

\section{Genetische Ursachen und Therapie beim Alport-Syndrom}

\section{Zusammenfassung}

Bei der Typ IV Kollagen-Erkrankung AlportSyndrom (AS) handelt es sich um eine progressive hereditäre Nephropathie. Klinische Zeichen sind zunächst Hämaturie und Proteinurie, im weiteren Verlauf kommt es zu einem terminalen Nierenversagen. Zusätzlich werden extrarenale Manifestationen wie Innenohr-Schwerhörigkeit und Augenveränderungen beobachtet. Man unterscheidet drei Erbgänge: 85 \% der Fälle sind X-chromosomal, ca. $10 \%$ autosomal und weniger als $5 \%$ digenisch. Ursächlich sind Varianten in den Kollagen Typ IV-Genen COL4A3, COL4A4 (beide autosomal) und COL4A5 (X-chromosomal). Die Symptomatik heterozygoter Anlageträger wurde früher als benigne familiäre Hämaturie bezeichnet. Da Anlageträger jedoch häufig keinen benignen Verlauf zeigen, werden sie inzwischen auch unter der Diagnose „Alport-Syndrom" geführt.

Der Humangenetiker hat daher beim AS eine wichtige Lotsenfunktion: Bei früher Diagnose ist das AS inzwischen gut behandelbar, wodurch das terminale Nierenversagen um mehrere Jahre hinausgezögert und damit die Lebenserwartung verbessert werden kann. Aufgrund der Therapiemöglichkeiten sollte die (molekulargenetische) Diagnose bei Betroffenen, auch bei heterozygoten Anlageträgern, frühzeitig gestellt werden. Mit diesem Artikel sollen die genetischen Ursachen des AS, mögliche genetische Einflussfaktoren auf den variablen Phänotyp, die unterschiedlichen Krankheitsstadien, Komplikationen sowie die derzeit zugelassene Behandlung aufgezeigt werden, um eine bestmögliche lebenslange Betreuung des Patienten zu gewährleisten.

Schlüsselwörter

Alport-Syndrom · Benigne familiäre Hämaturie · Nierenfibrose · Nephroprotektive Therapie · Typ IV Kollagen

\section{Genetic causes and therapy in Alport Syndrome}

\section{Abstract}

Patients with the hereditary disease Alport syndrome (AS) develop progressive renal fibrosis due to variants in type IV collagen genes. In the first years of life, AS starts with hematuria and proteinuria, finally leading to end-stage renal disease and extrarenal symptoms such as hearing impairment and ocular changes. Variants in three different genes can cause AS, COL4A5 (X-chromosomal) in $85 \%, C O L 4 A 3$ or COL4A4 (autosomal) in $10 \%$, and digenic variants in less than $5 \%$ of the cases. In the past, the symptomatic form in patients with a heterozygous variant was classified as thin basement membrane disease or benign familial hematuria. However, patients with a heterozygous variant often have a non-benign disease course. Therefore, these patients are now also given the diagnosis "Alport syndrome." If diagnosed early, AS is treatable. Renal failure can be delayed by years and life expectancy can be improved. Because of the available treatment options, the molecular genetic diagnosis should be made as soon as possible in every affected child and in all patients with a heterozygous variant. Unfortunately, the diagnosis is often made too late during early adolescence.

This article serves as a guideline for the genetic background of AS, possible additional (genetic) modifiers, possible additional complications, and the current therapeutic approach for the optimal lifelong care of patients living with AS. For genetic experts, it is important to know that this nephroprotective approach begins with an early genetic diagnosis guiding the timeline of possible therapeutic interventions.

\section{Keywords}

Alport syndrome - Benign familial hematuria Renal fibrosis - Nephroprotective therapy . Type IV collagen 


\begin{tabular}{|c|c|c|c|c|}
\hline & Art der Studie & Einschlusskriterien & Rekrutierung & Studienende geplant \\
\hline $\begin{array}{l}\text { EARLY PRO-TECT } \\
\text { Alport } \\
\text { NCT01485978 }\end{array}$ & $\begin{array}{l}\text { Doppelblinde randomisierte Phase } \\
\text { 3-Interventionsstudie } \\
\text { Interventionen: Ramipril vs. Placebo } \\
\text { Haupt-Endpunkte: Medikamen- } \\
\text { tensicherheit, Fortschreiten der } \\
\text { Albuminurie }\end{array}$ & $\begin{array}{l}\text { Alter 2-17 Jahre } \\
\text { nur Kinder mit Vollbild des Alport- } \\
\text { Syndroms } \\
\text { nur Frühstadien: Mikrohä- } \\
\text { maturie, Mikroalbuminurie, GFR } \\
>90 \mathrm{ml} / \mathrm{min} / 1,73 \mathrm{~m}^{2}\end{array}$ & Beendet 9/2015 & $\begin{array}{l}\text { Beginn } 2012 \\
\text { Ende 08/2019 }\end{array}$ \\
\hline $\begin{array}{l}\text { HERA } \\
\text { NCT02855268 }\end{array}$ & $\begin{array}{l}\text { Doppelblinde randomisierte Phase } \\
\text { 2-Interventionsstudie } \\
\text { Interventionen: anti-microRNA21 vs. } \\
\text { Placebo } \\
\text { Haupt-Endpunkte: eGFR-Verlust }\end{array}$ & $\begin{array}{l}\text { Alter } 16-60 \text { Jahre } \\
\text { alle Formen des Alport-Syndroms } \\
\text { Krankheitsstadien (unter Vor- } \\
\text { behalt): Proteinurie und GFR } \\
<90 \mathrm{ml} / \mathrm{min} / 1,73 \mathrm{~m}^{2} \text { GFR-Ver- } \\
\text { lust größer als } 4 \mathrm{ml} / \mathrm{min}^{\prime} / 1,73 \mathrm{~m}^{2} \mathrm{im} \\
\text { Jahr („fast progressors") }\end{array}$ & Seit 2017 & $\begin{array}{l}\text { Beginn } 05 / 2017 \\
\text { Ende geplant } 12 / 2020\end{array}$ \\
\hline $\begin{array}{l}\text { CARDINAL } \\
\text { NCT03019185 }\end{array}$ & $\begin{array}{l}\text { Doppelblinde randomisierte Phase } \\
\text { 2/3-Interventionsstudie } \\
\text { Interventionen: Bardoxolonmethyl } \\
\text { vs. Placebo } \\
\text { Haupt-Endpunkte: eGFR-Verlust }\end{array}$ & $\begin{array}{l}\text { Alter } 12-60 \text { Jahre } \\
\text { alle Formen des Alport-Syndroms } \\
\text { Krankheitsstadien (unter Vorbe- } \\
\text { halt): Mikroalbuminurie oder Pro- } \\
\text { teinurie, GFR }<90 \mathrm{ml} / \mathrm{min} / 1,73 \mathrm{~m}^{2}\end{array}$ & Seit 2017 & $\begin{array}{l}\text { Beginn 05/2017 } \\
\text { Ende geplant } 12 / 2020\end{array}$ \\
\hline $\begin{array}{l}\text { ATHENA } \\
\text { NCT02136862 }\end{array}$ & $\begin{array}{l}\text { Nicht interventionelle Beobach- } \\
\text { tungsstudie; prospektiv } \\
\text { beobachtete Interventionen: natürli- } \\
\text { cher Verlauf } \\
\text { Haupt-Endpunkte: eGFR-Verlauf }\end{array}$ & $\begin{array}{l}\text { Alter } 16-65 \text { Jahre } \\
\text { alle Formen des Alport-Syndroms } \\
\text { Krankheitsstadien: Proteinurie und } \\
\text { GFR }<90 \mathrm{ml} / \mathrm{min} / 1,73 \mathrm{~m}^{2}\end{array}$ & Beendet 2017 & $\begin{array}{l}\text { Beginn } 2015 \\
\text { Ende } 02 / 2018\end{array}$ \\
\hline $\begin{array}{l}\text { Europäisches Alport- } \\
\text { Therapieregister } \\
\text { NCT02378805 }\end{array}$ & $\begin{array}{l}\text { Nicht interventionelle Beobach- } \\
\text { tungsstudie; retrospektiv und teils } \\
\text { prospektiv } \\
\text { beobachtete Interventionen: ACE- } \\
\text { Hemmer, AT1-Antagonisten, Spiro- } \\
\text { nolacton, Statine, Paricalcitol } \\
\text { Haupt-Endpunkte: Dialysebeginn, } \\
\text { Tod }\end{array}$ & $\begin{array}{l}\text { Alter 0-99 Jahre } \\
\text { alle Formen des Alport-Syndroms } \\
\text { alle Krankheitsstadien einschließ- } \\
\text { lich Dialyse oder Transplantation }\end{array}$ & Offen bis 2038 & $\begin{array}{l}\text { Beginn } 2006 \\
\text { Ende geplant } 2038\end{array}$ \\
\hline
\end{tabular}

eine Orientierung für die Off-Label-Therapie, solange klinische Studien noch keine Entscheidungsgrundlage liefern. Jeder Patient sollte im Register ( $\bullet$ Tab. 2) erfasst werden, weil die Off-Label-Therapie ohne wissenschaftliche Auswertung als unethisch angesehen wird.

Im klinischen Alltag wird das Fortschreiten der Mikroalbuminurie zur Poteinurie oft mit Verzögerung festgestellt und die RAAS-Blockade auch erst verzögert eingeleitet. Bei Kindern mit Loss-ofFunction-Varianten kann man eine Therapie bereits ab dem Stadium der Mikroalbuminurie erwägen, bei allen anderen Patienten spätestens bei Auftreten der Proteinurie [36, 37]. Die Frage nach einem noch früheren Therapiebeginn wird die EARLY PRO-TECT Alport-Studie 2019 hoffentlich beantworten. Möglicherweise ist die Dialyse bei der Mehrzahl der Patienten mit MissenseVarianten vermeidbar. Von Brüderpaaren wissen wir, dass jedes Jahr, welches die Diagnose früher gestellt wird, dem Patienten mehrere zusätzliche Jahre mit erhaltener Nierenfunktion bringt [35].

\section{Mögliche genetische Modifier beim Alport-Syndrom}

Aktuell sind verschiedene NGS-Panels verfügbar, die insbesondere Varianten in Genen, die für Schlitzmembranproteine kodieren, analysieren. Auch wenn tierexperimentell erste Hinweise vorliegen, dass Polymorphismen und heterozygote Varianten in den Schlitzmembranproteinen den Krankheitsverlauf beim Alport-Syndrom beeinflussen, kann derzeit aufgrund unzureichender Datenlage noch keine abschließende genetische Bewertung dieser Varianten vorgenommen werden. In naher Zukunft wird deshalb die engere Zusammenarbeit zwischen einsendendem (Kinder-)Nephrologen und Humangenetiker noch bedeutender.

\section{Nephrologischer Leitfaden zur Therapie}

Patienten mit Alport-Syndrom: ACEHemmer ab Proteinurie $>0,3$ g/Tag, AT1Antagonisten sind second-line [20, 34, 38-40]. Empfohlen wird Ramipril bis $10 \mathrm{mg} /$ Tag bzw. $6 \mathrm{mg} / \mathrm{m}^{2}$. Kinder in frühen Stadien sollten nur in Studien wie EARLY PRO-TECT Alport behandelt werden [41].

Heterozygote Anlageträger mit TBMN: ACE-Hemmer bei volljährigen Patienten mit Mikroalbuminurie und bei allen Patienten mit Proteinurie [33, 36-38]. Auch Patienten mit Hämaturie sollten jährlich untersucht werden, insbesondere auf Risikofaktoren [39]. Heterozygote X-linked- oder ARAS-Anlageträger (mit TBMN) werden hier nicht unterschieden, denn beide haben ein - therapierbares erhöhtes renales Risiko! 


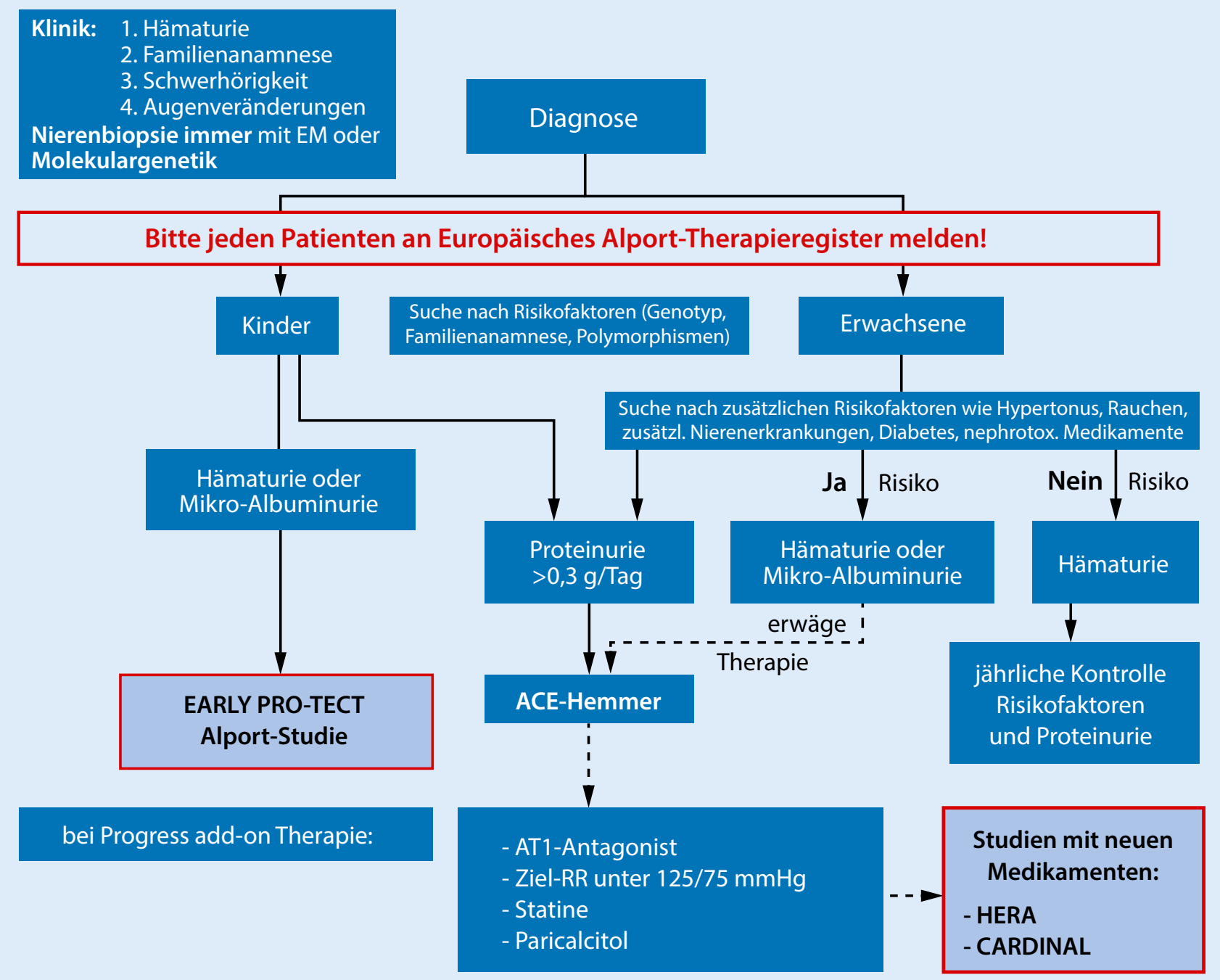

Abb. 1 \ Synopsis der Diagnose- und Therapieempfehlungen (nach [33]). Zur Diagnostik gehören neben der nephrologischen Standarddiagnostik eine vollständige Familienanamnese (einschließlich Untersuchung der Angehörigen auf Mikrohämaturie) und die Abklärung auf Innenohrschwerhörigkeit und Augenveränderungen (Spaltlampenuntersuchung)

\section{(4) Supportive Maßnahmen}

Die Pathogenese des Alport-Syndroms ist hochkompliziert, letztlich aber Folge eines simplen mechanischen Stabilitätsproblems der GBM. Die Effektivität von supportiven Maßnahmen beim AS zum Schutz der GBM wird unterschätzt. Studien an Jugendlichen mit AS lassen vermuten, dass sich rezidivierende bakterielle Infekte, schlechter Zahnstatus, hoher Salzkonsum, hoher Konsum von tierischem Eiweiß, extremer Kraftsport, Rauchen, Grenzwerthypertonie und Übergewicht negativ auf die GBM auswirken [20]. Eine Änderung der Lebensgewohnheiten verzögert wahrscheinlich das Nierenversagen um Jahre. Wir versuchen derzeit, dies im Tiermodell wissenschaftlich zu beweisen.

\section{(5) Therapieeskalation bei zunehmender Proteinurie}

Auch wenn ACE-Hemmer den Krankheitsverlauf um Jahre verzögern, schreitet die Erkrankung dennoch fort. Bei zunehmender Proteinurie werden in Deutschland in erster Linie AT1-Antagonisten additiv zum ACE-Hemmer eingesetzt. Bei steigendem Blutdruck und/ oder zunehmender Proteinurie können alle Antihypertensiva-Klassen verwendet werden, vorzugsweise langwirksame
Ca-Antagonisten, Diuretika und ggf. Spironolacton. Der Ziel-RR liegt bei unter $125 / 75 \mathrm{~mm} \mathrm{Hg}$. Ziele sind hier die Reduktion der Proteinurie und zu jedem Krankheitsstadium der Schutz der restlichen Glomeruli vor Hyperfiltration.

\section{(6) Therapiemöglichkeiten und -ziele bei Sekundärkomplikationen}

Prinzipiell entsprechen die Sekundärschäden bei AS denen von anderen Nierenerkrankungen. Speziell bei Erwachsenen mit Dyslipoproteinämie werden Statine aufgrund der im Alport-Tiermodell nachgewiesenen antifibrotischen Eigenschaften und bei Hyperparathy- 


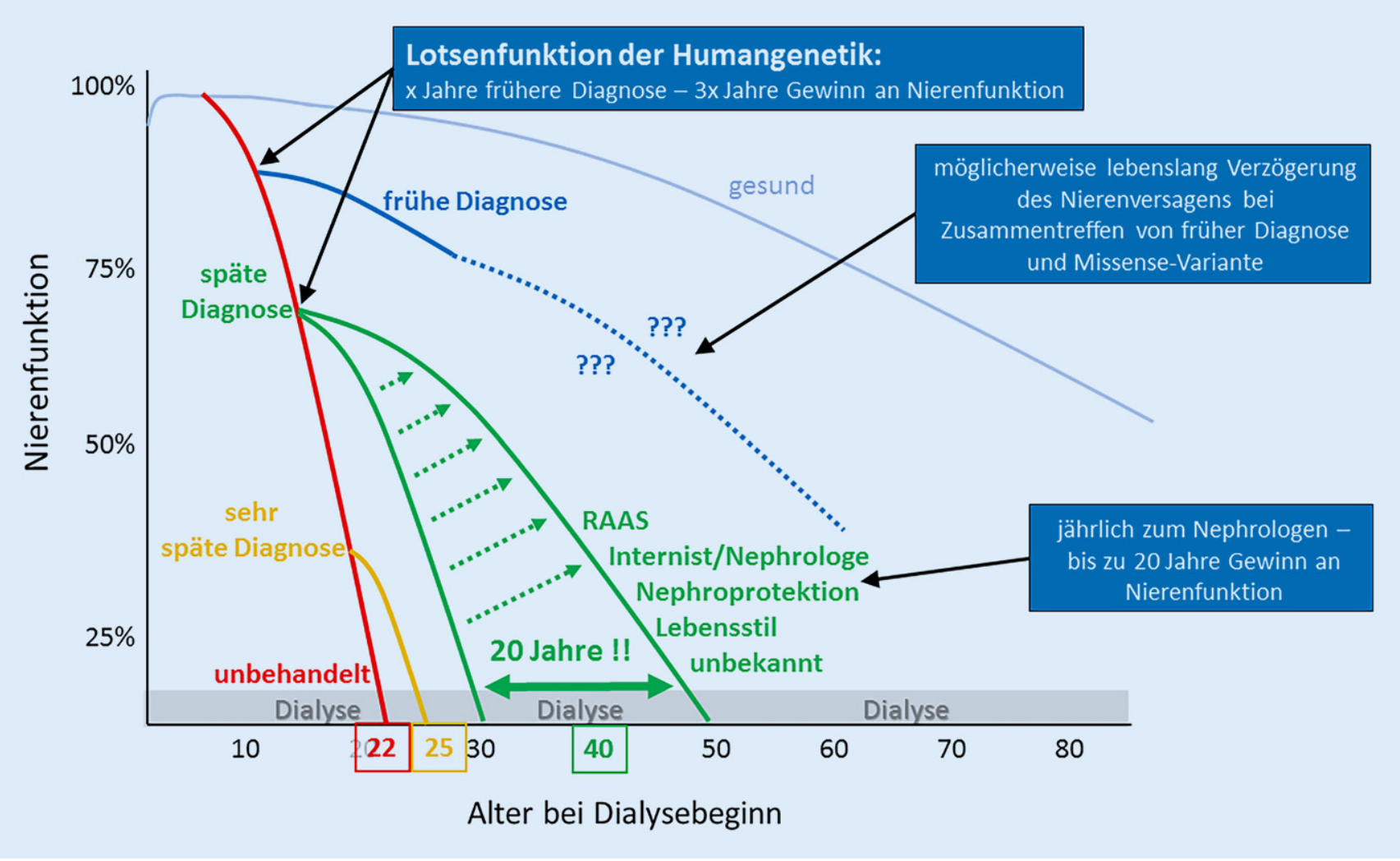

Abb. $2 \Delta$ Wissenschaftliche Rationale der überragenden Bedeutung der humangenetischen Familienuntersuchung beim Alport-Syndrom. Eine frühe humangenetische Diagnose verzögert das Nierenversagen um viele Jahre.

Rot Natürlicher Verlauf des Alport-Syndroms in Europa. Abhängig vom Genotyp muss der unbehandelte Patient im Median mit 22 Jahren an die Dialyse. Gelb Der Beginn der RAAS-Blockade bei einer glomerulären Filtrationsrate (GFR) $<60 \mathrm{ml} / \mathrm{min} / 1,73 \mathrm{~m}^{2}$ verzögert das Nierenversagen "nur“ noch um 3 Jahre. Grün Der Beginn der RAAS-Blockade bei proteinurischen Patienten mit normaler GFR verzögert die Dialyse im Median um 18 Jahre. Der nephroprotektive Effekt in dieser Gruppe ist stark abhängig von Umweltfaktoren: Am effektivsten scheint die Kombination von RAAS-Blockade, regelmäßiger Anbindung an einen Nephrologen mit strenger Einstellung aller zusätzlichen Risikofaktoren, gesunder Lebensstil und Nichtrauchen.

Blau 10 Jahre Registerarbeit deuten an, dass möglicherweise Patienten mit sehr früher Diagnose durch den Humangenetiker und "günstigerem“ Genotyp (Missense-Variante) bei optimaler Therapie und nephrologischer Betreuung in Zukunft kein terminales Nierenversagen mehr erleiden werden. Diese Gruppe könnte durch Therapien mit neuen Medikamenten noch deutlich anwachsen

reoidismus Paricalcitol empfohlen [32, 33]. Beide Maßnahmen verzögern möglicherweise auch beim Menschen das Nierenversagen um Monate bis wenige Jahre und verringern das kardiovaskuläre Risiko.

\section{Nephrologischer Leitfaden zu Nierenersatzverfahren, Transplantation und Prognose}

\section{(7) Besonderheiten bei Dialyse und bei Transplantation}

Die Lebenserwartung von Patienten mit AS unter Dialyse und nach Transplantation ist besser als von gleichaltrigen Dialysepatienten mit anderen Nierenerkrankungen [40]. Spendewilligen Anlageträgern mit einer heterozygoten Vari- ante in einem der $\mathrm{COL} 4 A-\mathrm{Gene}$ ist von der Nieren-Lebendspende abzuraten.

\section{Ausblick}

Die frühzeitige Off-Label-Therapie mit ACE-Hemmern bei Kindern mit AS ist medizinischer Standard. Über die Studienzentrale kann jedem Patienten mit AS die Teilnahme an weltweiten klinischen Studien angeboten werden, die in den nächsten Jahren weitere Therapiemöglichkeiten aufzeigen werden. Das Ziel zukünftiger Studien ist nicht nur der Beleg des klinischen Nutzens, sondern insbesondere auch die Sicherheit neuer Medikamente. Dem Humangenetiker kommt hier eine wichtige Lotsenfunktion $\mathrm{zu}$, indem die frühe molekulargenetische Diagnosesicherung nicht nur zur umfassen- den Familienuntersuchung führt, sondern auch zur frühen organprotektiven Therapie.

\section{Fazit für die Praxis}

- Auch bei heterozygoten Anlageträgern (früher: benigne familiäre Hämaturie) sollte der Befund des Humangenetikers auf das erhöhte Risiko einer progredienten Nierenerkrankung hinweisen. Ebenso wie das AS ist auch die benigne familiäre Hämaturie nicht benigne. Beide Erkrankungen benötigen eine lebenslange nephrologische Betreuung und Therapie.

- Kernprobleme beim AS sind die mechanische Stabilität der GBM, Fehlsignale der Kollagenrezeptoren, 
der Podozyt mit lokalem RAAS und interstitielle Sekundärschäden.

- Vielen Patienten mit AS können aktuell klinische Interventionsstudien mit neuen Medikamenten angeboten werden.

- Die frühe Diagnose durch den Humangenetiker in Zusammenarbeit mit dem (Kinder-)Nephrologen ist entscheidend und verbessert durch die frühere RAAS-Blockade die Prognose um viele Jahre.

- Optimaler Therapiebeginn, Stufentherapie und supportive Maßnahmen haben bei vielen Patienten das Potenzial, einen dialysepflichtigen Erkrankungsverlauf zu vermeiden. Bitte weisen Sie Ihre Patienten in Ihrem genetischen Befund auf die Behandlungsmöglichkeiten hin.

\section{Korrespondenzadresse}

\section{Prof. Dr. Oliver Gross}

Klinik für Nephrologie und Rheumatologie, Universitätsmedizin Göttingen

Robert-Koch Str. 40, 37075 Göttingen,

Deutschland

gross.oliver@med.uni-goettingen.de

\section{PD Dr. Julia Hoefele}

Institut für Humangenetik,

Klinikum rechts der Isar,

Technische Universität München

Trogerstr. 32, 81675 München, Deutschland julia.hoefele@tum.de

Förderung. Die Universitätsmedizin Göttingen erhält Aufwandsentschädigungen von Reata Pharmaceuticals und Regulus Therapeutics für die Beratertätigkeit von 0. Gross. Die Arbeiten wurden bzw. werden unterstützt durch die DFG GR 1852/4-1, 4-2 und 6-1, das NIH, die Alport Syndrome Foundation und Kidney Foundation of Canada, die Deutsche Nierenstiftung, die KfH-Stiftung Präventivmedizin, die Französische Gesellschaft für erbliche Nierenerkrankungen (AIRG) und die Alport-Selbsthilfe e. V. Die GPN-gestützte EARLY PRO-TECT Alport-Studie wird gefördert durch das BMBF/DFG-Programm „Klinische Studien" (01KG1104), Sanofi-Aventis Deutschland GmbH stellt die Prüfmedikation kostenlos zur Verfügung.

\section{Einhaltung ethischer Richtlinien}

Interessenkonflikt. O. Gross und J. Hoefele geben an, dass kein Interessenkonflikt besteht.

Dieser Beitrag beinhaltet keine von den Autoren durchgeführten Studien an Menschen oder Tieren.
Open Access. Dieser Artikel wird unter der Creative Commons Namensnennung 4.0 International Lizenz (http://creativecommons.org/licenses/by/4.0/deed. de) veröffentlicht, welche die Nutzung, Vervielfältigung, Bearbeitung, Verbreitung und Wiedergabe in jeglichem Medium und Format erlaubt, sofern Sie den/die ursprünglichen Autor(en) und die Quelle ordnungsgemäßnennen, einen Link zur Creative Commons Lizenz beifügen und angeben, ob Änderungen vorgenommen wurden.

\section{Literatur}

1. Weber S, Strasser K, Rath S, Kittke A, Beicht $S$, Alberer $M$, Lange-Sperandio $B$, Hoyer PF, Benz MR, Ponsel S, Weber LT, Klein HG, Hoefele $J$ (2016) Identification of 47 novel mutations in patients with Alport syndrome and thin basement membrane nephropathy. Pediatr Nephrol 31:941-955

2. Kashtan CE, Ding J, Garosi G, Heidet L, Massella L, Nakanishi K, Nozu K, Renieri A, Rheault M, Wang F, Gross $O$ (2018) Alport syndrome: a unified classification of genetic disorders of collagen IV alpha345: a position paper of the Alport Syndrome Classification Working Group. Kidney Int 93:1045-1051

3. Savige J, Ariani F, Mari F, Bruttini M, Renieri A, Gross O, Deltas C, Flinter F, Ding J, Gale DP, Nagel M, Yau M, Shagam L, Torra R, Ars E, Hoefele J, Garosi G, Storey H (2018) Expert consensus quidelines for the genetic diagnosis of Alport syndrome. Pediatr Nephrol. https://doi.org/10.1007/s00467018-3985-4

4. Fallerini $C$, Baldassarri $M$, Trevisson $E$, Morbidoni V, La Manna A, Lazzarin R, Pasini A, Barbano G, Pinciaroli AR, Garosi G, Frullanti E, Pinto AM, Mencarelli MA, Mari F, Renieri A, Ariani F (2017) Alport syndrome: impact of digenic inheritance in patients management. Clin Genet 92:34-44

5. Mencarelli MA, Heidet L, Storey $H$, van Geel M, Knebelmann B, Fallerini C, Miglietti N, Antonucci MF, Cetta F, Sayer JA, van den Wijngaard A, Yau S, Mari F, Bruttini M, Ariani F, Dahan K, Smeets B, Antignac C, Flinter F, Renieri A (2015) Evidence of digenic inheritance in Alport syndrome. J Med Genet 52:163-174

6. Gross O, Netzer KO, Lambrecht R, Seibold S, Webe M (2002) Meta-analysis of genotype-phenotype correlation in X-linked Alport syndrome: impact on clinical counselling. Nephrol Dial Transplant 17:1218-1227

7. Bekheirnia MR, Reed B, Gregory MC, McFann K, Shamshirsaz AA, Masoumi A, Schrier RW (2010) Genotype-phenotype correlation in X-linked Alport syndrome. J Am Soc Nephrol 21:876-883

8. Rheault MN (2012) Women and Alport syndrome Pediatr Nephrol 27:41-46

9. Savige J, Colville D, Rheault M, Gear S, Lennon R, Lagas S, Finlay M, Flinter F (2016) Alport syndrome in women and girls. Clin J Am Soc Nephrol 11:1713-1720

10. Jais JP, Knebelmann B, Giatras I, De Marchi $M$, Rizzoni G, Renieri A, Weber M, Gross O, Netzer KO, Flinter F, Pirson $Y$, Dahan $K$, Wieslander J, Persson U, Tryggvason K, Martin P, Hertz JM, Schroder C, Sanak M, Carvalho MF, Saus J, Antignac C, Smeets H, Gubler MC (2003) $\mathrm{X}$-linked Alport syndrome: natural history and genotype-phenotype correlations in girls and women belonging to 195 families: a „European Community Alport Syndrome Concerted Action" study. J Am Soc Nephrol 14:2603-2610
11. Vetrie D, Flinter F, Bobrow M, Harris A (1992) X inactivation patterns in females with Alport's syndrome: a means of selecting against a deleterious gene? J Med Genet 29:663-666

12. Rheault MN, Kren SM, Hartich LA, Wall M, Thomas W, Mesa HA, Avner P, Lees GE, Kashtan CE, Segal $Y(2010) X$-inactivation modifies disease severity in female carriers of murine $X$-linked Alport syndrome. Nephrol Dial Transplant 25:764-769

13. Kashtan CE (2003) Alport syndrome and thin basement membrane Nephropathy: diseases arising from mutations in type IV collagen. Saudi J Kidney Dis Transpl 14:276-289

14. Papazachariou L, Demosthenous $\mathrm{P}$, Pieri $\mathrm{M}, \mathrm{Pa}$ pagregoriou G, Savva I, Stavrou C, Zavros M, Athanasiou Y, loannou K, Patsias C, Panagides A, Potamitis C, Demetriou K, Prikis M, Hadjigavriel M, Kkolou M, Loukaidou P, Pastelli A, Michael A, Lazarou A, Arsali M, Damianou L, Goutziamani I, Soloukides A, Yioukas L, Elia A, Zouvani I, Polycarpou P, Pierides A, Voskarides K, Deltas C (2014) Frequency of COL4A3/COL4A4 mutations amongst families segregating glomerular microscopic hematuria and evidence for activation of the unfolded protein response. Focal and segmental glomerulosclerosis is a frequent development during ageing. PLoS ONE 9:e115015

15. Gale DP (2013) How benign is hematuria? Using genetics to predict prognosis. Pediatr Nephrol 28:1183-1193

16. Hempel M, Haack TB, Eck S, Prokisch H (2011) Next generation sequencing. Monatsschr Kinderheilkd 159:827-833

17. Cosgrove D, Meehan DT, Grunkemeyer JA, Kornak JM, Sayers R, Hunter WJ, Samuelson GC (1996) Collagen COL4A3 knockout: a mouse model for autosomal Alport syndrome. Genes Dev 10:2981-2992

18. Beirowski B, Weber M, Gross O (2006) Chronic renal failure and shortened lifespan in COL4A3+/-mice: an animal model for thin basement membrane nephropathy. J Am Soc Nephrol 17:1986-1994

19. Gross O, Beirowski B, Koepke ML, Kuck J, Reiner M, Addicks K, Smyth N, Schulze-Lohoff E, Weber M (2003) Preemptive ramipril therapy delays renal failure and reduces renal fibrosis in COL4A3knockout mice with Alport syndrome. Kidney Int 63:438-446

20. Gross O, Perin L, Deltas C (2014) Alport syndrome from bench to bedside: the potential of current treatment beyond RAAS blockade and the horizon of future therapies. Nephrol Dial Transplant 29(Suppl4):iv124-iv130

21. Rubel D, Stock J, Ciner $A$, Hiller $H$, Girgert $R$, Muller GA, Gross $O$ (2014) Antifibrotic, nephroprotective effects of paricalcitol versus calcitriol on top of ACEinhibitor therapy in the COL4A3 knockout mouse model for progressive renal fibrosis. Nephrol Dial Transplant 29:1012-1019

22. Kruegel J, Rubel D, Gross O (2013) Alport syndrome - insights from basic and clinical research. Nat Rev Nephrol 9:170-178

23. Gomez IG, MacKenna DA, Johnson BG, Kaimal $V$, Roach AM, Ren S, Nakagawa N, Xin C, Newitt R, Pandya S, Xia TH, Liu X, Borza DB, Grafals $M$, Shankland SJ, Himmelfarb J, Portilla D, Liu S, Chau BN, Duffield JS (2015) Anti-microRNA21 oligonucleotides prevent Alport nephropathy progression by stimulating metabolic pathways. JClin Invest 125:141-156

24. de Zeeuw D, Akizawa T, Audhya P, Bakris GL, Chin M, Christ-Schmidt H, Goldsberry A, Houser M, Krauth M, Lambers Heerspink HJ, McMurray JJ, Meyer CJ, Parving HH, Remuzzi G, Toto RD, Vaziri ND, Wanner 
C, Wittes J, Wrolstad D, Chertow GM, Investigators BT (2013) Bardoxolone methyl in type 2 diabetes and stage 4 chronic kidney disease. N Engl J Med 369:2492-2503

25. Girgert R, Martin M, Kruegel J, Miosge N, Temme J, Eckes B, Muller GA, Gross O (2010) Integrin alpha2deficient mice provide insights into specific functions of collagen receptors in the kidney. Fibrogenesis Tissue Repair 3:19

26. Gross O, Girgert R, Beirowski B, Kretzler M, Kang HG, Kruegel J, Miosge N, Busse AC, Segerer S, Vogel WF, Muller GA, Weber M (2010) Loss of collagenreceptor DDR1 delays renal fibrosis in hereditary type IV collagen disease. Matrix Biol 29:346-356

27. Rubel $D$, Frese J, Martin $M$, Leibnitz $A$, Girgert R, Miosge N, Eckes B, Muller GA, Gross O (2014) Collagen receptors integrin alpha2beta1 and discoidin domain receptor 1 regulate maturation of the glomerular basement membrane and loss of integrin alpha2beta1 delays kidney fibrosis in COL4A3 knockout mice. Matrix Biol 34:13-21

28. Borza CM, Su Y, Tran TL, Yu L, Steyns N, Temple KJ, Skwark MJ, Meiler J, Lindsley CW, Hicks BR, Leitinger B, Zent R, Pozzi A (2017) Discoidin domain receptor 1 kinase activity is required for regulating collagen IV synthesis. Matrix Biol 57-58:258-271

29. Heikkila P, Tryggvason K, Thorner P (2000) Animal models of Alport syndrome: advancing the prospects for effective human gene therapy. Exp Nephrol 8:1-7

30. Ninichuk V, Gross O, Segerer S, Hoffmann R, Radomska E, Buchstaller A, Huss R, Akis N, Schlondorff D, Anders HJ (2006) Multipotent mesenchymal stem cells reduce interstitial fibrosis but do not delay progression of chronic kidney disease in collagen4A3-deficient mice. Kidney Int 70:121-129

31. Gross O, Borza DB, Anders HJ, Licht C, Weber M, Segerer S, Torra R, Gubler MC, Heidet L, Harvey S, Cosgrove D, Lees G, Kashtan C, Gregory M, Savige J, Ding J, Thorner P, Abrahamson DR, Antignac C, Tryggvason K, Hudson B, Miner JH (2009) Stem cell therapy for Alport syndrome: the hope beyond the hype. Nephrol Dial Transplant 24:731-734

32. Miner JH, Baigent $C$, Flinter $F$, Gross $O$, Judge $P$, Kashtan CE, Lagas S, Savige J, Blatt D, Ding J, Gale DP, Midgley JP, Povey S, Prunotto M, Renault D Skelding J, Turner AN, Gear S (2014) The 2014 International Workshop on Alport Syndrome. Kidney Int 86:679-684

33. Gross O, Kashtan CE, Rheault MN, Flinter F, Savige J, Miner JH, Torra R, Ars E, Deltas C, Savva I, Perin L, Renieri A, Ariani F, Mari F, Baigent C, Judge $P$, Knebelman $B$, Heidet $L$, Lagas $S$, Blatt D, Ding J, Zhang Y, Gale DP, Prunotto M, Xue Y, Schachter AD, Morton LCG, Blem J, Huang M, Liu S, Vallee S, Renault D, Schifter J, Skelding J, Gear S, Friede T, Turner AN, Lennon R (2017) Advances and unmet needs in genetic, basic and clinical science in Alport syndrome: report from the 2015 International Workshop on Alport Syndrome. Nephrol Dial Transplant 32:916-924

34. Stock J, Kuenanz J, Glonke N, Sonntag J, Frese J, Tonshoff B, Hocker B, Hoppe B, Feldkotter M, Pape L, Lerch C, Wygoda S, Weber M, Muller GA, Gross O (2017) Prospective study on the potential of RAAS blockade to halt renal disease in Alport syndrome patients with heterozygous mutations. Pediatr Nephrol 32:131-137

35. Gross O, Licht C, Anders HJ, Hoppe B, Beck B, Tonshoff B, Hocker B, Wygoda S, Ehrich JH, Pape L, Konrad M, Rascher W, Dotsch J, MullerWiefel DE, Hoyer P, Study Group Members of the Gesellschaft fur Padiatrische N, Knebelmann B,
Pirson $Y$, Grunfeld JP, Niaudet $P$, Cochat $P$, Heidet L, Lebbah S, Torra R, Friede T, Lange K, Muller GA, Weber M (2012) Early angiotensin-converting enzyme inhibition in Alport syndrome delays renal failure and improves life expectancy. Kidney Int 81:494-501

36. Kashtan CE, Ding J, Gregory M, Gross $\mathrm{O}$, Heidet L, Knebelmann B, Rheault M, Licht C (2013) Clinical practice recommendations for the treatment of Alport syndrome: a statement of the Alport Syndrome Research Collaborative. Pediatr Nephrol 28:5-11

37. Savige J, Gregory M, Gross O, Kashtan C, Ding J, Flinter F (2013) Expert guidelines for the management of Alport syndrome and thin basement membrane nephropathy. J Am Soc Nephrol 24:364-375

38. Temme J, Peters F, Lange $K$, Pirson $Y$, Heidet $L$, Torra R, Grunfeld JP, Weber M, Licht C, Muller GA, Gross O (2012) Incidence of renal failure and nephroprotection by RAAS inhibition in heterozygous carriers of X-chromosomal and autosomal recessive Alport mutations. Kidney Int 81:779-783

39. Nagel M, Gross $O$ (2016) Das Alport-Syndrom als wichtige Differentialdiagnose einer Hämaturie. Nieren-und Hochdruckkrankheiten 45:26-272

40. Temme J, Kramer A, Jager KJ, Lange K, Peters F, Muller GA, Kramar R, Heaf JG, Finne P, Palsson R, Reisaeter AV, Hoitsma AJ, Metcalfe W, Postorino M, Zurriaga O, Santos JP, Ravani P, Jarraya F, Verrina E, Dekker FW, Gross O (2012) Outcomes of male patients with Alport syndrome undergoing renal replacement therapy. Clin J Am Soc Nephrol 7:1969-1976

41. Gross O, Friede T, Hilgers R, Gorlitz A, Gavenis K, Ahmed R, Durr U (2012) Safety and Efficacy of the ACE-Inhibitor Ramipril in Alport Syndrome: The Double-Blind, Randomized, Placebo-Controlled, Multicenter Phase III EARLY PRO-TECT Alport Tria in Pediatric Patients. ISRN Pediatr 2012:436046 